Pacific

Journal of

Mathematics

\title{
KNOT ADJACENCY, GENUS AND ESSENTIAL TORI
}

EFSTRATIA KALFAGIANNI AND XIAO-SONG LiN 


\title{
KNOT ADJACENCY, GENUS AND ESSENTIAL TORI
}

\author{
Efstratia Kalfagianni AND XiAO-SONG LiN
}

\begin{abstract}
A knot $K$ is called $\boldsymbol{n}$-adjacent to another $\operatorname{knot} \boldsymbol{K}^{\prime}$ if $\boldsymbol{K}$ admits a projection containing $n$ generalized crossings such that changing any $0<m \leq n$ of them yields a projection of $K^{\prime}$. We apply techniques from the theory of sutured 3-manifolds, Dehn surgery and the theory of geometric structures of 3-manifolds to study the extent to which nonisotopic knots can be adjacent to each other. A consequence of our main result is that if $K$ is $\boldsymbol{n}$-adjacent to $K^{\prime}$ for all $n \in \mathbb{N}$, then $K$ and $K^{\prime}$ are isotopic. This provides a partial verification of the conjecture of $V$. Vassiliev that finite type knot invariants distinguish all knots. We also show that if no twist about a crossing circle $L$ of a knot $K$ changes the isotopy class of $K$, then $L$ bounds a disc in the complement of $K$. This leads to a characterization of nugatory crossings on knots.
\end{abstract}

\section{Introduction}

A crossing disc for a knot $K \subset S^{3}$ is an embedded disc $D \subset S^{3}$ such that $K$ intersects int $D$ twice with zero algebraic number. Let $q \in \mathbb{Z}$. Performing $\frac{1}{q}$ surgery on $L_{1}:=\partial D_{1}$, changes $K$ to another knot $K^{\prime} \subset S^{3}$. We say that $K^{\prime}$ is obtained from $K$ by a generalized crossing change of order $q$ (see Figure 1).

An $n$-collection for a knot $K$ is a pair $(\mathscr{D}, \boldsymbol{q})$, such that

(i) $\mathscr{D}:=\left\{D_{1}, \ldots, D_{n}\right\}$ is a set of disjoint crossing discs for $K$,

(ii) $\boldsymbol{q}:=\left\{\frac{1}{q_{1}}, \ldots, \frac{1}{q_{n}}\right\}$, with $q_{i} \in \mathbb{Z} \backslash\{0\}$, and

(iii) the knots $L_{1}:=\partial D_{1}, \ldots, L_{n}:=\partial D_{n}$ are labeled by $\frac{1}{q_{1}}, \ldots, \frac{1}{q_{n}}$.

The link $L:=\bigcup_{i=1}^{n} L_{i}$ is called the crossing link associated to $(\mathscr{D}, \boldsymbol{q})$.

We will use the notation

$$
\boldsymbol{i}:=\left(i_{1}, \ldots, i_{n}\right) \in\{0,1\}^{n}
$$

MSC2000: 57M25, 57M27, 57M50.

Keywords: knot adjacency, essential tori, finite type invariants, Dehn surgery, sutured 3-manifolds,

Thurston norm, Vassiliev's conjecture.

Kalfagianni was supported in part by NSF grants DMS-0104000 and DMS-0306995 and by a grant through the Institute for Advanced Study. Lin was supported in part by the Overseas Youth Cooperation Research Fund of NSFC and by NSF grants DMS-0102231 and DMS-0404511. 


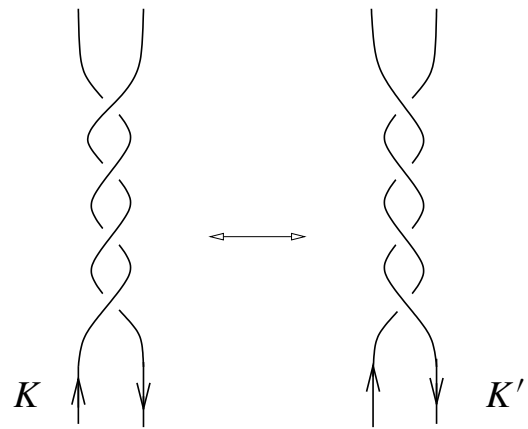

Figure 1. The knots $K$ and $K^{\prime}$ differ by a generalized crossing change of order $q=-4$.

for an $n$-tuple with $i_{j} \in\{0,1\}$ for $j=1, \ldots, n$. We also set $\mathbf{0}:=(0, \ldots, 0)$, $\mathbf{1}:=(1, \ldots, 1)$, and $\boldsymbol{i}_{j}:=(0, \ldots, 0,1,0, \ldots, 0)$ with the nonzero entry at the $j$-th place.

Given a knot $K$ and an $n$-collection $(\mathscr{D}, \boldsymbol{q})$, for every $\boldsymbol{i}$, denote by $K(\boldsymbol{i})$ the knot obtained from $K$ by a surgery modification of order $q_{j}$ along each $L_{j}$ for which $i_{j}=1$, and of order 0 along each $L_{j}$ for which $i_{j}=0$.

Definition 1.1. $K$ is $n$-adjacent to $K^{\prime}$ if there exists an $n$-collection $(\mathscr{D}, \boldsymbol{q})$ for $K$ such that the knot $K(\boldsymbol{i})$ is isotopic to $K^{\prime}$ for every $\boldsymbol{i} \neq \mathbf{0}$. In this situation we write $K \stackrel{n}{\longrightarrow} K^{\prime}$ and we say that $(\mathscr{D}, \boldsymbol{q})$ transforms $K$ to $K^{\prime}$.

Our main result is this:

Theorem 1.2. Suppose that $K$ and $K^{\prime}$ are nonisotopic knots. There exists a constant $C\left(K, K^{\prime}\right)$ such that if $K \stackrel{n}{\longrightarrow} K^{\prime}$, then $n \leq C\left(K, K^{\prime}\right)$.

The quantity $C\left(K, K^{\prime}\right)$ can be expressed in terms of computable invariants of the knots $K$ and $K^{\prime}$. Let $g(K)$ and $g\left(K^{\prime}\right)$ denote the genera of $K$ and $K^{\prime}$ and let $g:=\max \left\{g(K), g\left(K^{\prime}\right)\right\}$. The constant $C\left(K, K^{\prime}\right)$ encodes information about the relative size of $g(K), g\left(K^{\prime}\right)$ and the behavior of the satellite structures of $K$ and $K^{\prime}$ under the Dehn surgeries imposed by knot adjacency. In many cases $C\left(K, K^{\prime}\right)$ can be made explicit. For example, when $g(K)>g\left(K^{\prime}\right)$ we have $C\left(K, K^{\prime}\right)=6 g-3$. Thus, in this case, Theorem 1.2 can be restated as follows:

Theorem 1.3. Suppose that $K, K^{\prime}$ are knots with $g(K)>g\left(K^{\prime}\right)$. If $K \stackrel{n}{\longrightarrow} K^{\prime}$, then $n \leq 6 g(K)-3$.

In the case that $K^{\prime}$ is the trivial knot Theorem 1.3 was proved by H. Howards and J. Luecke [2002].

A crossing of a knot $K$, with crossing disc $D$, is called nugatory if and only if $\partial D$ bounds a disc that is disjoint from $K$. The techniques used in the proof of Theorem 1.2 have applications to the question of whether a crossing change that 
doesn't change the isotopy class of the underlying knot is nugatory [Kirby 1997, Problem 1.58]. As a corollary of the proof of Theorem 1.2 we obtain the following characterization of nugatory crossings:

Corollary 1.4. For a crossing disc $D$ of a knot $K$ let $K(r)$ denote the knot obtained by a twist of order $r$ along $D$. The crossing is nugatory if and only if $K(r)$ is isotopic to $K$ for all $r \in \mathbb{Z}$.

Definition 1.1 is equivalent to the definition of $n$-adjacency given in [Kalfagianni and Lin 2004b] (and in the abstract of this paper). With this reformulation, it follows that $n$-adjacency implies $n$-similarity in the sense of [Ohyama 1990], which in turn, as shown in [Ng and Stanford 1999], implies $n$-equivalence. Gussarov showed that two knots are $n$-equivalent precisely when all of their finite type invariants of orders $<n$ are the same. Vassiliev [1990] has conjectured that if two oriented knots have all of their finite type invariants the same then they are isotopic. In the light of Gussarov's result, this conjecture can be reformulated as follows:

Conjecture 1.5 (Vassiliev). Suppose that $K$ and $K^{\prime}$ are knots that are $n$-equivalent for all $n \in \mathbb{N}$. Then $K$ is isotopic to $K^{\prime}$.

Theorem 1.2 implies the following corollary, which provides a partial verification to Vassiliev's conjecture:

Corollary 1.6. If $K \stackrel{n}{\longrightarrow} K^{\prime}$ for all $n \in \mathbb{N}$, then $K$ and $K^{\prime}$ are isotopic.

We now describe the contents of the paper and the idea of the proof of the main theorem. Let $K$ be a knot and let $(\mathscr{D}, \boldsymbol{q})$ be a $n$-collection with associated crossing link $L$. Since the linking number of $K$ and every component of $L$ is zero, $K$ bounds a Seifert surface in the complement of $L$. Thus, we can define the genus of $K$ in the complement of $L$, say $g_{L}^{n}(K)$. In Section 2 we study the extent to which a Seifert surface of $K$ that is of minimal genus in the complement of $L$ remains of minimal genus under various surgery modifications along the components of $L$. Using a result of from [Gabai 1987] we show that if $K \stackrel{n}{\longrightarrow} K^{\prime}$, and $(\mathscr{D}, \boldsymbol{q})$ is an $n$-collection that transfers $K$ to $K^{\prime}$ then $g_{L}^{n}(K)=g:=\max \left\{g(K), g\left(K^{\prime}\right)\right\}$, where $g(K), g\left(K^{\prime}\right)$ denotes the genus of $K, K^{\prime}$ respectively. This is done in Theorem 2.1.

In Section 3 we prove Theorem 1.3. In Section 4, we finish the proof of Theorem 1.2: We begin by defining a notion of $m$-adjacency between knots $K, K^{\prime}$ with respect to an one component crossing link $L_{1}$ of $K$ (see Definition 4.1). To describe our approach in more detail, set $N:=S^{3} \backslash \eta\left(K \cup L_{1}\right)$, and let $\tau(N)$ denote the number of disjoint, pairwise nonparallel, essential embedded tori in $N$. We employ results of Cooper and Lackenby [1998], Gordon [1998] and McCullough [2006] and an induction argument on $\tau(N)$ to show the following: Given knots $K, K^{\prime}$, there exists a constant $b\left(K, K^{\prime}\right) \in \mathbb{N}$ such that if $K$ is $m$-adjacent to $K^{\prime}$ with respect to a crossing link $L_{1}$ then either $m \leq b\left(K, K^{\prime}\right)$ or $L_{1}$ bounds an embedded disc 
in the complement of $K$. This is done in Theorem 4.3. Theorem 2.1 implies that if $K \stackrel{n}{\longrightarrow} K^{\prime}$ and $n>m(6 g-3)$, then an $n$-collection that transforms $K$ to $K^{\prime}$ gives rise to a crossing link $L_{1}$ such that $K$ is $m$-adjacent to $K^{\prime}$ with respect to $L_{1}$. Combining this with Theorem 4.3 yields Theorem 1.2.

In Section 5, we present some applications of the results of Section 4 and the methods used in their proofs. Also, for every $n \in \mathbb{N}$, we construct examples of nonisotopic knots $K, K^{\prime}$ such that $K \stackrel{n}{\longrightarrow} K^{\prime}$.

Throughout the entire paper we work in the PL or the smooth category. In [Kalfagianni 2006], the techniques of this paper are refined and used to study adjacency to fibered knots and the problem of nugatory crossings in fibered knots. In [Kalfagianni and Lin 2004a] the results of this paper are used to obtain criteria for detecting nonfibered knots and for detecting the nonexistence of symplectic structures on certain 4-manifolds. Further applications include, in the same paper, constructions of 3-manifolds that are indistinguishable by certain Cochran-Melvin finite type invariants, and in [Kalfagianni 2004] constructions of hyperbolic knots with trivial Alexander polynomial and arbitrarily large volume.

\section{Taut surfaces, knot genus and multiple crossing changes}

Let $K$ be a knot and $(\mathscr{D}, \boldsymbol{q})$ an $n$-collection for $K$ with associated crossing link $L$. Since the linking number of $K$ and every component of $L$ is zero, $K$ bounds a Seifert surface $S$ in the complement of $L$. Define

$$
g_{n}^{L}(K):=\min \{\operatorname{genus}(\mathrm{S}) \mid S \text { a Seifert surface of } K \text { as above }\} .
$$

Our main result in this section is the following:

Theorem 2.1. Suppose that $K \stackrel{n}{\longrightarrow} K^{\prime}$, for some $n \geq 1$. Let $(\mathscr{D}, \boldsymbol{q})$ be an $n$ collection that transforms $K$ to $K^{\prime}$ with associated crossing link $L$. We have

$$
g_{n}^{L}(K)=\max \left\{g(K), g\left(K^{\prime}\right)\right\} .
$$

In particular, $g_{n}^{L}(K)$ is independent of $L$ and $n$.

Before we prove this we need some preparation. For a link $\bar{L} \subset S^{3}$ we will use $\eta(\bar{L})$ to denote a regular neighborhood of $\bar{L}$. For a knot $K \subset S^{3}$ and an $n$-collection $(\mathscr{D}, \boldsymbol{q})$, let

$$
M_{L}:=S^{3} \backslash \eta(K \cup L),
$$

where $L$ is the crossing link associated to $(\mathscr{D}, \boldsymbol{q})$.

Lemma 2.2. Suppose that $K, K^{\prime}$ are knots such that $K \stackrel{n}{\longrightarrow} K^{\prime}$ for some $n \geq 1$. Let $(\mathscr{D}, \boldsymbol{q})$ be an $n$-collection that transforms $K$ to $K^{\prime}$. If $M_{L}$ is reducible then a component of $L$ bounds an embedded disc in the complement of $K$. In particular, $K$ is isotopic to $K^{\prime}$. 
Proof. Let $\Sigma$ be an essential 2-sphere in $M_{L}$. Assume that $\Sigma$ has been isotoped so that the intersection $I:=\Sigma \cap\left(\bigcup_{i=1}^{n} D_{i}\right)$ is minimal. Notice that we must have $I \neq \varnothing$ since otherwise $\Sigma$ would bound a 3-ball in $M_{L}$. Let $c \in\left(\Sigma \cap D_{i}\right)$ denote a component of $I$ that is innermost on $\Sigma$; that is $c$ bounds a disc $E \subset \Sigma$ such that int $E \cap\left(\bigcup_{i=1}^{n} D_{i}\right)=\varnothing$. Since $\Sigma$ is separating in $M_{L}$, the disc $E$ can't contain just one point of $K \cap D_{i}$. Also $E$ can't be disjoint from $K$ or $c$ could be removed by isotopy. Hence $E$ contains both points of $K \cap D_{i}$ and so $c=\partial E$ is parallel to $\partial D_{i}$ in $D_{i} \backslash K$. It follows that $L_{i}$ bounds an embedded disc in the complement of $K$. Since $\frac{1}{q_{i}}$-surgery on $L_{i}$ turns $K$ into $K^{\prime}$, we conclude that $K$ is isotopic to $K^{\prime}$.

Definition 2.3 [Thurston 1986]. Let $M$ be a compact, oriented 3-manifold with boundary $\partial M$. For a compact, connected, oriented surface $(S, \partial S) \subset(M, \partial M)$, the complexity $\chi^{-}(S)$ is defined by

$\chi^{-}(S):=\max \{0,-\chi(S)\}$, where $\chi(S)$ denotes the Euler characteristic of $S$. If $S$ is disconnected then $\chi^{-}(S)$ is defined to be the sum of the complexities of all the components of $S$. Let $\eta(\partial S)$ denote a regular neighborhood of $\partial S$ in $\partial M$. The Thurston norm $x(z)$ of a homology class $z \in H_{2}(M, \eta(\partial S))$ is the minimal complexity over all oriented, embedded surfaces representing $z$. The surface $S$ is called taut if it is incompressible and we have $x([S, \partial S])=\chi^{-}(S)$; that is $S$ is norm-minimizing.

The proof of the next lemma follows from the definitions:

Lemma 2.4. Let $(\mathscr{D}, \boldsymbol{q})$ be an $n$-collection for a knot $K$ with associated crossing link $L$ and $M_{L}:=S^{3} \backslash \eta(K \cup L)$. A compact, connected, oriented surface $(S, \partial S) \subset$ $\left(M_{L}, \partial \eta(K)\right)$, such that $\partial S=K$, is taut if and only if among all Seifert surfaces of $K$ in the complement of $L, S$ has the minimal genus.

For $\boldsymbol{i} \in\{0,1\}^{n}$ as above, let $M_{L}(\boldsymbol{i})$ denote the 3-manifold obtained from $M_{L}$ by performing Dehn filling on $\partial M_{L}$ with slope $\frac{1}{q_{j}}$ for the components $\partial \eta\left(L_{j}\right)$ for which $i_{j}=1$, and slope $\infty:=\frac{1}{0}$ for the components where $i_{j}=0$. Clearly we have $M_{L}(\boldsymbol{i})=S^{3} \backslash \eta(K(\boldsymbol{i}))$, where $K(\boldsymbol{i})$ is as in Definition 1.1. Also let $M_{L}^{+}(\boldsymbol{i})$ and $M_{L}^{-}(i)$ denote the 3-manifolds obtained from $M_{L}$ by only performing Dehn filling with slope $\frac{1}{q_{j}}$ and $\infty$, respectively, on the components $\partial \eta\left(L_{j}\right)$ for which $i_{j}=1$.

Lemma 2.5. Let $(\mathscr{D}, \boldsymbol{q})$ be an $n$-collection for a knot $K$ such that $M_{L}$ is irreducible. Let $(S, \partial S) \subset\left(M_{L}, \partial \eta(K)\right)$ be an oriented surface with $\partial S=K$ that is taut. Then at least one of $M_{L}^{+}\left(\boldsymbol{i}_{j}\right), M_{L}^{-}\left(\boldsymbol{i}_{j}\right)$ is irreducible and $S$ remains taut in that 3-manifold.

(The notation $\boldsymbol{i}_{j}$ is defined on page 252.)

Proof. The proof uses a result of [Gabai 1987] in the spirit of [Scharlemann and Thompson 1989]: For $j \in\{1, \ldots, n\}$ set $M^{+}:=M_{L}^{+}\left(\boldsymbol{i}_{j}\right)$ and $M^{-}:=M_{L}^{-}\left(\boldsymbol{i}_{j}\right)$. Also set $L^{j}:=L \backslash L_{j}$ and $T_{j}:=\partial \eta\left(L_{j}\right)$. We distinguish two cases: 
Case 1: Suppose that every embedded torus that is incompressible in $M_{L}$ and it separates $L^{j} \cup S$ from $L_{j}$, is parallel to $T_{j}$. Then $M_{L}$ is $S_{L_{j}}$-atoroidal (see Definition 1.6 of [Gabai 1987]). By Corollary 2.4 of the same reference, there is at most one Dehn filling along $T_{j}$ that yields a 3-manifold which is either reducible or in which $S$ doesn't remain taut. Thus the desired conclusion follows.

Case 2: There exists an embedded torus $T \subset M_{L}$ such that (i) $T$ is incompressible in $M_{L}$; (ii) $T$ separates $L^{j} \cup S$ from $L_{j}$; and (iii) $T$ is not parallel to $T_{j}$. In $S^{3}, T$ bounds a solid torus $V$, with $\partial V=T$. Suppose, for a moment, that $L_{j}$ lies in int $V$ and $L^{j} \cup S$ lies in $S^{3} \backslash V$. If $V$ is knotted in $S^{3}$ then, since $L_{j}$ is unknotted, $L_{j}$ is homotopically inessential in $V$. But then $T$ compresses in $V$ and thus in $M_{L}$; a contradiction. If $V$ is unknotted in $S^{3}$ then the longitude of $V$ bounds a disc $E$ in $S^{3} \backslash V$. Since $S$ is disjoint from $T, K$ intersects $E$ at least twice. At the same time, since $T$ is incompressible in $M_{L}$ and $K$ intersects $D_{j}$ twice, $L_{j}$ is isotopic to the core of $V$. Hence, $T$ is parallel to $T_{j}$ in $M_{L}$; a contradiction. Hence $L^{j} \cup S$ lies in int $V$ while $L_{j}$ lies in $S^{3} \backslash V$. We will show that $M^{+}, M^{-}$are irreducible and that $S$ remains taut in both of these 3-manifolds.

Among all tori in $M_{L}$ that have properties (i)-(iii) stated above, choose $T$ to be one that minimizes $\left|T \cap D_{j}\right|$. Then $D_{j} \cap T$ consists of a single curve which bounds a disc $D^{*} \subset$ int $D_{j}$, such that $\left(K \cap D_{j}\right) \subset$ int $D^{*}$ and $D^{*}$ is a meridian disc of $V$. See Figure 2.

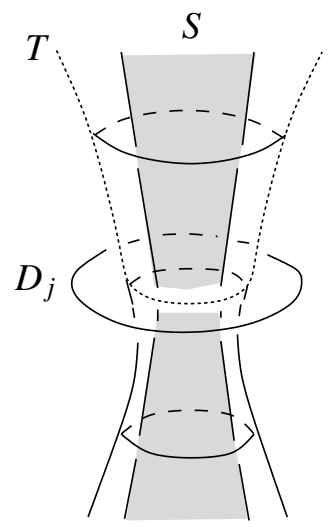

Figure 2. The intersection of $T$ and $S$ with $D_{j}$.

Since $T$ is not parallel to $T_{j}, V$ must be knotted. For $r \in \mathbb{Z}$, let $M(r)$ denote the 3-manifold obtained from $M_{L}$ by performing Dehn filling along $\partial \eta\left(L_{j}\right)$ with slope $\frac{1}{r}$. Since the core of $V$ intersects $D_{j}$ once, the Dehn filling doesn't unknot $V$ and $T=\partial V$ remains incompressible in $M(r) \backslash V$. On the other hand, $T$ is incompressible in $V \backslash\left(K \cup L^{j}\right)$ by definition. Notice that both $M(r) \backslash V$ and 
$V \backslash\left(K \cup L^{j}\right)$ are irreducible and

$$
M(r)=(M(r) \backslash V) \bigcup_{T}\left(V \backslash\left(K \cup L^{j}\right)\right) .
$$

We conclude that $T$ remains incompressible in $M(r)$ and $M(r)$ is irreducible. In particular $M^{+}$and $M^{-}$are both irreducible.

Next we show that $S$ remains taut in $M^{+}$and $M^{-}$. By Lemma 2.4, we must show that $S$ is a minimal genus surface for $K$ in $M^{+}$and in $M^{-}$. To that end, let $S_{1}$ be a minimal genus surface for $K$ in $M^{+}$or in $M^{-}$. We may isotope so that $S_{1} \cap T$ is a collection of parallel essential curves on $T$. Since the linking number of $K$ and $L_{j}$ is zero, $S_{1} \cap T$ is homologically trivial in $T$. Thus, we may attach annuli along the components of $S_{1} \cap T$ and then isotope off $T$ in int $V$, to obtain a Seifert surface $S_{1}^{\prime}$ for $K$ that is disjoint from $L_{j}$. Thus $S_{1}^{\prime}$ is a surface in the complement of $L$. Since $T$ is incompressible, no component of $S_{1} \backslash V$ is a disc. Thus, genus $\left(S_{1}^{\prime}\right) \leq \operatorname{genus}\left(S_{1}\right)$. On the other hand, by the definition of $S$, $\operatorname{genus}(S) \leq \operatorname{genus}\left(S_{1}^{\prime}\right)$ and thus genus $(\mathrm{S}) \leq \operatorname{genus}\left(S_{1}\right)$.

Lemma 2.6. Let $(\mathscr{D}, \boldsymbol{q})$ be an $n$-collection for a knot $K$ such that $M_{L}$ is irreducible. Let $(S, \partial S) \subset\left(M_{L}, \partial \eta(K)\right)$ be an oriented surface with $\partial S=K$ that is taut. There exists at least one sequence $i:=\left(i_{1}, \ldots i_{n}\right)$, with $i_{j} \in\{1,0\}$, such that $S$ remains taut in $M_{L}(\boldsymbol{i})$. Thus $g(K(\boldsymbol{i}))=\operatorname{genus}(S)$.

Proof. The proof is by induction on $n$. For $n=1$, the conclusion follows from Lemma 2.5. Suppose the conclusion is true for every $m<n$ and every $m$-collection $\left(\mathscr{D}_{1}, \boldsymbol{q}_{1}\right)$ of a knot $K_{1}$ such that $M_{L^{1}}$ is irreducible, where $L^{1}$ denotes the crossing link associated to $\mathscr{D}_{1}$ and $M_{L^{1}}:=S^{3} \backslash \eta\left(K_{1} \cup L^{1}\right)$.

Let $K,(\mathscr{D}, \boldsymbol{q})$ and $S$ be as in the statement of the lemma. By Lemma 2.5, at least one of $M_{L}^{ \pm}\left(\boldsymbol{i}_{1}\right)$, say $M_{L}^{-}\left(\boldsymbol{i}_{1}\right)$, is irreducible and $S$ remains taut in that 3-manifold. Let

$$
\mathscr{D}_{1}:=\left\{D_{2}, \ldots, D_{n}\right\} \quad \text { and } \quad \boldsymbol{q}_{1}:=\left\{q_{2}, \ldots, q_{n}\right\} .
$$

Let $L^{1}:=L \backslash L_{1}$ and let $K_{1}$ denote the image of $K$ in $M_{L}^{-}\left(\boldsymbol{i}_{1}\right)$. Clearly, $M_{L^{1}}=$ $M_{L}^{-}\left(i_{1}\right)$ and thus $M_{L^{1}}$ is irreducible. By the induction hypothesis, applied to $K_{1}$ and the $(n-1)$-collection $\left(\mathscr{D}_{1}, \boldsymbol{q}_{1}\right)$, it follows that there is at least one sequence $\hat{\boldsymbol{\imath}}:=\left(\hat{\imath}_{2}, \ldots \hat{\imath}_{n}\right) \in\{0,1\}^{n-1}$ such that $S$ remains taut in $M_{L^{1}}(\hat{\boldsymbol{\imath}})$. Since $M_{L^{1}}(\hat{\boldsymbol{\imath}})=$ $M_{L}(\boldsymbol{i})$, where $\boldsymbol{i}:=\left(0, \hat{\imath}_{2}, \ldots \hat{\imath}_{n}\right)$, the desired conclusion follows.

Proof of Theorem 2.1. Suppose $K \stackrel{n}{\longrightarrow} K^{\prime}$ and let $L$ and $M_{L}$ be as in the statement of the theorem. Let $S$ be a Seifert surface for $K$ in the complement of $L$ such that genus $(S)=g_{n}^{L}(K)$. First, assume that $M_{L}$ is irreducible. By Lemma 2.4, $S$ gives rise to a surface $(S, \partial S) \subset\left(M_{L}, \eta(\partial S)\right)$ that is taut. By Lemma 2.6, there exists at least one sequence $i \in\{0,1\}^{n}$ such that $S$ remains taut in $M_{L}(i)$. There are three cases to consider, depending on the relation between $g(K)$ and $g\left(K^{\prime}\right)$. 
If $g(K)>g\left(K^{\prime}\right)$, for every $\boldsymbol{i} \neq \mathbf{0}$, we have

$$
g\left(K^{\prime}\right)=g(K(\boldsymbol{i}))<g(K) \leq \operatorname{genus}(S) .
$$

Therefore $S$ doesn't remain taut in $M_{L}(\boldsymbol{i})=S^{3} \backslash \eta(K(\boldsymbol{i}))$. Hence $S$ must remain taut in $M_{L}(\mathbf{0})=S^{3} \backslash \eta(K)$ and we have $g_{n}^{L}(K)=g(K)$.

If $g(K)<g\left(K^{\prime}\right)$, we have a $n$-collection $\left(\mathscr{D}^{\prime}, \boldsymbol{q}^{\prime}\right)$ for $K^{\prime}$ where $\mathscr{D}^{\prime}=\mathscr{D}$ and $\boldsymbol{q}^{\prime}=-\boldsymbol{q}$, such that $K^{\prime}(\boldsymbol{i})=K^{\prime}$ for all $\boldsymbol{i} \neq \mathbf{1}$ and $K^{\prime}(\mathbf{1})=K$. So we may argue similarly as in case (1) that $g_{n}^{L}(K)=g\left(K^{\prime}\right)$. In fact, in this case, $S$ must remain taut in $M_{L}(\boldsymbol{i})$ for all $\boldsymbol{i} \neq \mathbf{0}$.

Finally, if $g(K)=g\left(K^{\prime}\right), S$ remains taut in $M_{L}(i)$ for all $\boldsymbol{i}$, and it follows that $g_{n}^{L}(K)=g\left(K^{\prime}\right)=g(K)$.

Now suppose that $M_{L}$ is reducible. By Lemma 2.2, there is at least one component of $L$ that bounds an embedded disc in the complement of $K$. Let $L^{1}$ denote the union of the components of $L$ that bound disjoint discs in the complement of $K$ and let $L^{2}:=L \backslash L^{1}$. We may isotope $S$ so that it is disjoint from the discs bounded by the components of $L^{1}$. Now $S$ can be viewed as taut surface in $M_{L^{2}}:=S^{3} \backslash \eta\left(K \cup L^{1}\right)$. If $L^{2}=\varnothing$, the conclusion is clearly true. Otherwise $M_{L^{2}}$ is irreducible and the argument described above applies.

\section{Genus reducing $\boldsymbol{n}$-collections}

The purpose of this section is to prove Theorem 1.2 in the case that $g(K)>g\left(K^{\prime}\right)$. The argument is essentially that in the proof of the main result of [Howards and Luecke 2002].

Proof of Theorem 1.3. Let $K, K^{\prime}$ be as in the statement of the theorem. Let $(\mathscr{D}, \boldsymbol{q})$ be an $n$-collection that transforms $K$ to $K^{\prime}$ with associated crossing link $L$. Let $S$ be a Seifert surface for $K$ that is of minimum genus among all surfaces bounded by $K$ in the complement of $L$. By Theorem 2.1 we have genus $(S)=g(K)$. Since $S$ is incompressible, after an isotopy, we can arrange so that for $i=1, \ldots, n$, each closed component of $S \cap$ int $D_{i}$ is essential in $D_{i} \backslash K$ and thus parallel to $L_{i}=\partial D_{i}$ on $D_{i}$. Then, after an isotopy of $L_{i}$ in the complement of $K$, we may assume that $S \cap$ int $D_{i}$ consists of a single properly embedded arc $\left(\alpha_{i}, \partial \alpha_{i}\right) \subset(S, \partial S)$ (Figure 3 ). Notice that $\alpha_{i}$ is essential on $S$. For, otherwise, $D_{i}$ would bound a disc in the complement of $K$ and thus the genus of $K$ could not be lowered by surgery on $L_{i}$.

We claim that no two of the arcs $\alpha_{1}, \ldots \alpha_{n}$, can be parallel on $S$. For suppose to the contrary that the $\operatorname{arcs} \alpha_{i}:=$ int $D_{i} \cap S$ and $\alpha_{j}:=$ int $D_{j} \cap S$ are parallel on $S$. Then the crossing circles $L_{i}$ and $L_{j}$ cobound an embedded annulus that is disjoint from $K$. Let

$$
M:=S^{3} \backslash \eta\left(K \cup L_{i}\right) \quad \text { and } \quad M_{1}:=S^{3} \backslash \eta\left(K \cup L_{i} \cup L_{j}\right) .
$$




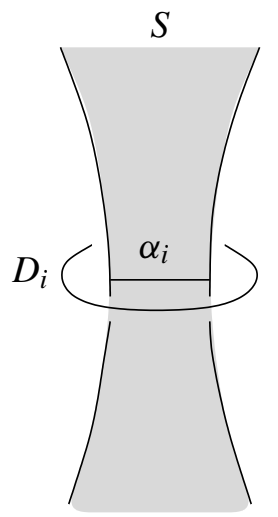

Figure 3. The intersection of $S$ with int $D_{i}$.

For $r, s \in \mathbb{Z}$ let $M(r)$ be the 3-manifold obtained from $M$ by filling in $\partial \eta\left(L_{i}\right)$ with slope $\frac{1}{r}$, and let $M_{1}(r, s)$ be the manifold obtained from $M_{1}$ by filling in $\partial \eta\left(L_{i} \cup L_{j}\right)$ with slopes $\frac{1}{r}$ and $\frac{1}{s}$. By assumption, $S$ doesn't remain taut in any $M\left(q_{i}\right)$ or $M_{1}\left(q_{i}, q_{j}\right)$. Since $L_{i}, L_{j}$ are coannular we see that $M_{1}\left(q_{i}, q_{j}\right)=M\left(q_{i}+\right.$ $q_{j}$ ). Notice that $q_{i}+q_{j} \neq q_{i}$ since otherwise we would conclude that a twist of order $q_{j}$ along $L_{j}$ cannot reduce the genus of $K$. Hence we would have two distinct Dehn fillings of $M$ along $\partial \eta\left(L_{i}\right)$ under which $S$ doesn't remain taut, contradicting Corollary 2.4 of [Gabai 1987]. Therefore, we conclude that no two of the arcs $\alpha_{1}, \ldots \alpha_{n}$, can be parallel on $S$. Now the conclusion follows since a Seifert surface of genus $g$ contains $6 g-3$ essential arcs no pair of which is parallel.

\section{Knot adjacency and essential tori}

In this section we complete the proof of Theorem 1.2. For this we need to study the case of $n$-adjacent knots $K \stackrel{n}{\longrightarrow} K^{\prime}$ in the special situation where all the crossing changes from $K$ to $K^{\prime}$ are supported on a single crossing circle of $K$. Using Theorem 2.1, we will see that the general case is reduced to this special one.

Knot adjacency with respect to a crossing circle. We begin with a refined version of the knot adjacency notion:

Definition 4.1. Let $K, K^{\prime}$ be knots and let $D_{1}$ be a crossing disc for $K$. We will say that $K$ is $m$-adjacent to $K^{\prime}$ with respect to the crossing circle $L_{1}:=\partial D_{1}$, if there exist nonzero integers $s_{1}, \ldots, s_{m}$ such that the following is true: For every nonempty $J \subset\{1, \ldots, m\}$, the knot obtained from $K$ by a surgery modification of order $s_{J}:=\sum_{j \in J} s_{j}$ along $L_{1}$ is isotopic to $K^{\prime}$. We will write

$$
K \stackrel{m, L_{1}}{\longrightarrow} K^{\prime} .
$$


Suppose that $K \stackrel{m, L_{1}}{\longrightarrow} K^{\prime}$ and consider the $m$-collection obtained by taking $m$ parallel copies of $D_{1}$ and labeling the $i$-th copy of $L_{1}$ by $\frac{1}{s_{i}}$. As follows immediately from the definitions, this $m$-collection transforms $K$ to $K^{\prime}$ in the sense of Definition 1.1; thus $K \stackrel{m}{\longrightarrow} K^{\prime}$. Here is a converse that's needed for the proof of Theorem 1.2:

Lemma 4.2. Let $K, K^{\prime}$ be knots and set $g:=\max \left\{g(K), g\left(K^{\prime}\right)\right\}$. Suppose that $K \stackrel{n}{\longrightarrow} K^{\prime}$. If $n>m(6 g-3)$ for some $m>0$, there exists a crossing link $L_{1}$ for $K$ such that $K \stackrel{m+1, L_{1}}{\longrightarrow} K^{\prime}$.

Proof. Let $(\mathscr{D}, \boldsymbol{q})$ be an $n$-collection that transforms $K$ to $K^{\prime}$ and let $L$ denote the associated crossing link. Let $S$ be a Seifert surface for $K$ that is of minimal genus among all surfaces bounded by $K$ in the complement of $L$. Isotope so that, for $i=1, \ldots, n$, the intersection $S \cap \operatorname{int} D_{i}$ is an $\operatorname{arc} \alpha_{i}$ that is properly embedded and essential on $S$. By Theorem 2.1, we have genus $(S)=g$. Since $n>m(6 g-3)$, the set $\left\{\alpha_{i} \mid i=1, \ldots, n\right\}$ contains at least $m+1$ arcs that are parallel on $S$. Suppose, without loss of generality, that these are the $\operatorname{arcs} \alpha_{i}, i=1, \ldots, m+1$. It follows that the components $L_{1}, \ldots, L_{m+1}$ of $L$ are isotopic in the complement of $K$; thus any surgery along any of these components can be realized as surgery on $L_{1}$. It now follows from Definitions 1.1 and 4.1 that $K \stackrel{m+1, L_{1}}{\longrightarrow} K^{\prime}$.

The main ingredient needed to complete the proof of Theorem 1.2 is provided by the following theorem:

Theorem 4.3. Given knots $K, K^{\prime}$, there exists a constant $b\left(K, K^{\prime}\right) \in \mathbb{N}$ such that if $L_{1}$ is a crossing circle of $K$ and $K \stackrel{m, L_{1}}{\longrightarrow} K^{\prime}$, then either $m \leq b\left(K, K^{\prime}\right)$ or $L_{1}$ bounds an embedded disc in the complement of $K$.

Proof of Theorem 1.2 assuming Theorem 4.3. Suppose that $K, K^{\prime}$ are nonisotopic knots with $K \stackrel{n}{\longrightarrow} K^{\prime}$. If $g(K)>g\left(K^{\prime}\right)$ the conclusion follows from Theorem 1.3 by simply taking $C\left(K, K^{\prime}\right):=6 g-3$. In general, let

$$
C\left(K, K^{\prime}\right):=b\left(K, K^{\prime}\right)(6 g-3),
$$

where $b:=b\left(K, K^{\prime}\right)$ is the constant of Theorem 4.3. We claim that $n \leq C\left(K, K^{\prime}\right)$. Suppose, to the contrary, that $n>C\left(K, K^{\prime}\right)$. By Lemma 4.2, there exists a crossing circle $L_{1}$ for $K$ such that $K \stackrel{b+1, L_{1}}{\longrightarrow} K^{\prime}$. By Theorem 4.3, $L_{1}$ bounds an embedded disc in the complement of $K$. But this implies that $K$ is isotopic to $K^{\prime}$ contrary to our assumption.

The rest of this section will be devoted to the proof of Theorem 4.3. For that we need to study whether the complement of $K \cup L_{1}$ contains essential tori and how these tori behave under the crossing changes from $K$ to $K^{\prime}$. Given $K, K^{\prime}$ and $L_{1}$ such that $K \stackrel{m, L_{1}}{\longrightarrow} K^{\prime}$, set $N:=S^{3} \backslash \eta\left(K \cup L_{1}\right)$ and $N^{\prime}:=S^{3} \backslash \eta\left(K^{\prime}\right)$. By assumption, $N^{\prime}$ is obtained by Dehn filling along the torus $T_{1}:=\partial \eta\left(L_{1}\right)$. If $N$ is reducible, 
Lemma 2.2 implies that $L_{1}$ bounds a disc in the complement of $K$; thus Theorem 4.3 holds. For irreducible $N$, as it turns out, there are three basic cases to consider:

(a) $K^{\prime}$ is a composite knot.

(b) $N$ is atoroidal.

(c) $N$ is toroidal and $K^{\prime}$ is not a composite knot.

By [Thurston 1979], if $N$ is atoroidal it is either hyperbolic (it admits a complete hyperbolic metric of finite volume) or it is a Seifert fibered space. To handle the hyperbolic case we will use a result of Cooper and Lackenby [1998]. The Seifert fibered spaces that occur are known to be very special and this case is handled by a case-by-case analysis. Case (c) is handled by induction on the number of essential tori contained in $N$. To set up this induction one needs to study the behavior of these essential tori under the Dehn fillings from $N$ to $N^{\prime}$. In particular, one needs to know the circumstances under which these Dehn fillings create essential tori in $N^{\prime}$. For this step, we will employ a result of Gordon [1998].

Composite knots. Here we examine the circumstances under which a knot $K$ is $n$-adjacent to a composite knot $K^{\prime}$. We will need the following theorem.

Theorem 4.4 [Torisu 1999]. Let $K^{\prime}:=K_{1}^{\prime} \# K_{2}^{\prime}$ be a composite knot and $K^{\prime \prime}$ a knot obtained from $K^{\prime}$ by a generalized crossing change with corresponding crossing disc $D$. If $K^{\prime \prime}$ is isotopic to $K^{\prime}$ then either $\partial D$ bounds a disc in the complement of $K^{\prime}$ or the crossing change occurs within $K_{1}^{\prime}$ or $K_{2}^{\prime}$.

Proof. For an ordinary crossing the result is [Torisu 1999, Theorem 2.1]. The proof given there works for generalized crossings.

The next lemma handles possibility (a) above ( $K^{\prime}$ is composite), reducing Theorem 4.3 to the case that $K^{\prime}$ is a prime knot.

Lemma 4.5. Let $K, K^{\prime}$ be knots such that $K \stackrel{m, L_{1}}{\longrightarrow} K^{\prime}$, where $L_{1}$ is a crossing circle for $K$. Suppose that $K^{\prime}:=K_{1}^{\prime} \# K_{2}^{\prime}$ is a composite knot. Then either $L_{1}$ bounds a disc in the complement of $K$ or $K$ is a connect sum $K=K_{1} \# K_{2}$ and there exist $J \in\left\{K_{1}, K_{2}\right\}$ and $J^{\prime} \in\left\{K_{1}^{\prime}, K_{2}^{\prime}\right\}$ such that $J \stackrel{m, L_{1}}{\longrightarrow} J^{\prime}$.

Proof. By assumption there is an integer $r \neq 0$ so that the knot $K^{\prime \prime}$ obtained from $K^{\prime}$ by a generalized crossing change of order $r$ is isotopic to $K^{\prime}$. By Theorem 4.4, either $L_{1}$ bounds a disc in the complement of $K^{\prime}$ or the crossing change occurs on one of $K_{1}^{\prime}, K_{2}^{\prime}$; say on $K_{1}^{\prime}$. Thus, in particular, in the latter case $L_{1}$ is a crossing link for $K_{1}^{\prime}$. Since $K$ is obtained from $K^{\prime}$ by twisting along $L_{1}, K$ is a, not necessarily nontrivial, connect sum of the form $K_{1} \# K_{2}^{\prime}$. By the uniqueness of knot decompositions it follows that $K_{1} \stackrel{m, L_{1}}{\longrightarrow} K_{1}^{\prime}$. 
Dehn surgeries that create essential tori. Suppose $M$ is a compact orientable 3manifold. For a collection $\mathscr{T}$ of disjointly embedded, pairwise nonparallel, essential tori in $M$ we will use $|\mathscr{T}|$ to denote the number of components of $\mathscr{T}$. By Haken's finiteness theorem [Hempel 1976, Lemma 13.2], the number

$$
\tau(M)=\max \{|\mathscr{T}| \mid \mathcal{T} \text { is a collection of tori as above }\}
$$

is well defined. A collection $\mathscr{T}$ for which $\tau(M)=|\mathscr{T}|$ will be called a Haken system.

We will study the behavior of essential tori under the various Dehn fillings from $N:=S^{3} \backslash \eta\left(K \cup L_{1}\right)$ to $N^{\prime}:=S^{3} \backslash \eta\left(K^{\prime}\right)$. Since $N^{\prime}$ is obtained from $N$ by Dehn filling along $T_{1}:=\partial \eta\left(L_{1}\right)$, essential tori in $N^{\prime}$ occur in two ways:

Type I: An essential torus $T^{\prime} \subset N^{\prime}$ that can be isotoped in $N \subset N^{\prime}$; thus such a torus is the image of an essential torus $T \subset N$.

Type II: An essential torus $T^{\prime} \subset N^{\prime}$ that is the image of an essential punctured torus $(P, \partial P) \subset\left(N, T_{1}\right)$, such that each component of $\partial P$ is parallel on $T_{1}$ to the curve along which the Dehn filling from $N$ to $N^{\prime}$ is done.

We begin with a lemma that examines circumstances under which twisting a knot that is geometrically essential inside a knotted solid torus $V$ yields a knot that is geometrically inessential inside $V$. In the notation of Definition 4.1, the lemma implies that an essential torus in $N$ either remains essential in $N\left(s_{J}\right)$, for all nonempty $J \subset\{1, \ldots, m\}$, or it becomes inessential in all $N\left(s_{J}\right)$.

Lemma 4.6. Let $V \subset S^{3}$ be a knotted solid torus and let $K_{1} \subset V$ be a knot that is geometrically essential in $V$. Let $D \subset$ int $V$ be a crossing disc for $K_{1}$ and let $K_{2}$ be a knot obtained from $K_{1}$ by a nontrivial twist along $D$. Suppose that $K_{1}$ is isotopic to $K_{2}$ in $S^{3}$. Then $K_{2}$ is geometrically essential in $V$. Furthermore, if $K_{1}$ is not the core of $V$ then $K_{2}$ is not the core of $V$.

Proof. Suppose that $K_{2}$ is not geometrically essential in $V$. Then there is an embedded 3-ball $B \subset$ int $V$ that contains $K_{2}$. Since making crossing changes on $K_{2}$ doesn't change the homology class it represents in $V$, the winding number of $K_{1}$ in $V$ must be zero. Set $L:=\partial D$ and $N:=S^{3} \backslash \eta(K \cup L)$. Let $S$ be a Seifert surface for $K_{1}$ such that among all the surfaces bounded by $K_{1}$ in $N, S$ has minimum genus. As usual we isotope $S$ so that $S \cap D$ is an $\operatorname{arc} \alpha$ properly embedded on $S$. As in the proof of Theorem 2.1, $S$ gives rise to Seifert surfaces $S_{1}, S_{2}$ of $K_{1}, K_{2}$, respectively. Now $K_{1}$ can be recovered from $K_{2}$ by twisting $\partial S_{2}$ along $\alpha$.

Claim. $L$ can be isotoped inside $B$ in the complement of $K_{1}$.

Assume this for the moment. Since $K_{1}$ is obtained from $K_{2}$ by a generalized crossing change supported on $L$ it follows that $K_{1}$ lies in $B$. Since this contradicts 
our assumption that $K_{1}$ is geometrically essential in $V, K_{2}$ must be geometrically essential in $V$. To finish the proof of the lemma, assuming the claim, observe that if $K_{1}$ is not the core $C$ of $V$, then $C$ is a companion knot of $K_{1}$. If $K_{2}$ is the core of $V, C$ and $K_{1}$ are isotopic in $S^{3}$ which by [Schubert 1953] is impossible.

We now prove the claim. Since $K_{1}, K_{2}$ are isotopic in $S^{3}$ by Corollary 2.4 of [Gabai 1987] (as used in the proof of Theorem 2.1), we see that $S_{1}$ and $S_{2}$ are minimum genus surfaces for $K_{1}$ and $K_{2}$ in $S^{3}$. By assumption $\partial V$ is a nontrivial companion torus of $K_{1}$. Since the winding number of $K_{1}$ in $V$ is zero, the intersections $S_{1} \cap \partial V$ and $S_{2} \cap \partial V$ are homologically trivial in $\partial V$. Thus, for $i=1,2$, we may replace the components of $S_{i} \cap \overline{S^{3} \backslash V}$ with boundary parallel annuli in int $V$ to obtain a Seifert surface $S_{i}^{\prime}$ inside $V$. It follows that $S_{i} \cap \overline{S^{3} \backslash V}$ is a collection of annuli and $S_{i}^{\prime}$ is a minimum genus Seifert surface for $K_{i}$. Now $S_{2}^{\prime}$ is a minimum genus Seifert surface for $K_{2}$ such that $\alpha \subset S_{2}^{\prime}$. By assumption, $K_{2}$ lies inside $B$. Since $S_{2}^{\prime}$ is incompressible and $V$ is irreducible, $S_{2}^{\prime}$ can be isotoped in $B$ by a sequence of disc trading isotopies in int $V$. But this isotopy will also bring $\alpha$ inside $B$ and thus $L$.

Next we focus on the case that $N^{\prime}$ is toroidal and examine the circumstances under which $N^{\prime}$ contains type II tori.

Proposition 4.7. Let $K, K^{\prime}$ be knots such that $K^{\prime}$ is a nontrivial satellite but not composite. Suppose that $K \stackrel{m, L_{1}}{\longrightarrow} K^{\prime}$, where $L_{1}$ is a crossing circle for $K$ and let the notation be as in Definition 4.1. At least one of the following is true:

(a) $L_{1}$ bounds an embedded disc in the complement of $K$.

(b) For every nonempty $J \subset\{1, \ldots, m\}$, there is in $N\left(s_{J}\right)$ a Haken system that doesn't contain tori of type II.

(c) We have $m \leq 6$.

Proof. For $s \in \mathbb{Z}$, let $N(s)$ be the 3-manifold obtained from $N$ by Dehn filling along $T_{1}$ with slope $\frac{1}{s}$. Assume that $L_{1}$ doesn't bound an embedded disc in the complement of $K$ and that, for some nonempty $J_{1} \subset\{1, \ldots, m\}, N\left(s_{J_{1}}\right)$ admits a Haken system that contains tori of type II. We claim that, for every nonempty $J \subset\{1, \ldots, m\}, N\left(s_{J}\right)$ has such a Haken system. To see this, first assume that $N$ doesn't contain essential embedded tori. Then, since $N^{\prime}=N\left(s_{J}\right)$ and $K^{\prime}$ is a nontrivial satellite, the conclusion follows. Suppose that $N$ contains essential embedded tori. By Lemma 4.6 it follows that an essential torus in $N$ either remains essential in $N\left(s_{J}\right)$, for all nonempty $J \subset\{1, \ldots, m\}$, or it becomes inessential in all $N\left(s_{J}\right)$ as above. Thus the number of type I tori in a Haken system of $N\left(s_{J}\right)$ is the same for all $J$ as above. Thus, since we assume that $N\left(s_{J_{1}}\right)$ has a Haken system containing tori of type II, a Haken system of $N\left(s_{J}\right)$ must contain tori of type II, for every nonempty $J \subset\{1, \ldots, m\}$. We distinguish two cases: 
Case 1: Suppose that $s_{1}, \ldots, s_{m}>0$ or $s_{1}, \ldots, s_{m}<0$. Let $s:=\sum_{j=1}^{m} s_{j}$ and recall that we assumed that $N$ is irreducible. By our discussion above, both of $N\left(s_{1}\right), N(s)$ contain essential embedded tori of type II. By [Gordon 1998, Theorem 1.1], we must have

$$
\Delta\left(s, s_{1}\right) \leq 5,
$$

where $\Delta\left(s, s_{1}\right)$ denotes the geometric intersection on $T_{1}$ of the slopes represented by $\frac{1}{s_{1}}$ and $\frac{1}{s}$. Since $\Delta\left(s, s_{1}\right)=\left|\sum_{j=2}^{m} s_{j}\right|$, and $\left|s_{j}\right| \geq 1$, in order for $\Delta\left(s, s_{1}\right)$ to be at least 5 we must have $m-1 \leq 5$ or $m \leq 6$.

Case 2: Suppose that not all of $s_{1}, \ldots, s_{m}$ have the same sign. Suppose, without loss of generality, that $s_{1}, \ldots, s_{k}>0$ and $s_{k+1}, \ldots, s_{m}<0$. Let $s:=\sum_{j=1}^{k} s_{j}$ and $t:=\sum_{j=k+1}^{m} s_{j}$. Since both of $N(s), N(t)$ contain essential embedded tori of type II, by [Gordon 1998, Theorem 1.1]

$$
\Delta(s, t) \leq 5 .
$$

But $\Delta(t, s)=s-t=\sum_{j=1}^{m}\left|s_{j}\right|$. Thus, in order for $\Delta(s, t) \leq 5$ to be true, we must have $m \leq 5$. The result follows.

Proposition 4.7 and Lemma 4.5 yield:

Corollary 4.8. Let $K, K^{\prime}$ be knots and let $L_{1}$ be a crossing circle for $K$. Suppose that the 3-manifold $N$ contains no essential embedded torus and that $K \stackrel{m, L_{1}}{\longrightarrow} K^{\prime}$. If $K^{\prime}$ is a nontrivial satellite, then either $m \leq 6$ or $L_{1}$ bounds an embedded disc in the complement of $K$.

Hyperbolic and Seifert fibered manifolds. We now deal with the case that the manifold $N$ is atoroidal. As already mentioned, by Thurston's uniformization theorem for Haken manifolds [Thurston 1979], $N$ is either hyperbolic or a Seifert fibered manifold.

First we recall some terminology about hyperbolic 3-manifolds. Let $N$ be a hyperbolic 3-manifold with boundary and let $T_{1}$ a component of $\partial N$. In int $N$ there is a cusp, which is homeomorphic to $T_{1} \times[1, \infty)$, associated with the torus $T_{1}$. The cusp lifts to an infinite set, say $\mathcal{H}$, of disjoint horoballs in the hyperbolic space $\mathbb{H}^{3}$ which can be expanded so that each horoball in $\mathscr{H}$ has a point of tangency with some other. The image of these horoballs under the projection $\mathbb{H}^{3} \rightarrow$ int $N$ is the maximal horoball neighborhood of $T_{1}$. The boundary $\mathbb{R}^{2}$ of each horoball in $\mathscr{H}$ inherits a Euclidean metric from $\mathbb{M}^{3}$ which in turn induces a Euclidean metric on $T_{1}$. A slope $s$ on $T_{1}$ defines a primitive element in $\pi_{1}\left(T_{1}\right)$ corresponding to a Euclidean translation in $\mathbb{R}^{2}$. The length of $s$, denoted by $l(s)$, is the length of the corresponding translation vector.

Given a slope $s$ on $T_{1}$, let us use $N[s]$ to denote the manifold obtained from $N$ by Dehn filling along $T_{1}$ with slope $s$. We remind the reader that in the case that 
the slope $s$ is represented by $\frac{1}{s}$, for some $s \in \mathbb{Z}$, we use the notation $N(s)$ instead. Next we recall a result of Cooper and Lackenby the proof of which relies on work of Thurston and Gromov. We only state the result in the special case needed here:

Theorem 4.9 [Cooper and Lackenby 1998]. Let $N^{\prime}$ be a compact orientable manifold, with $\partial N^{\prime}$ a collection of tori. Let $N$ be a hyperbolic manifold and let $\mathbf{s}$ be a slope on a toral component $T_{1}$ of $\partial N$ such that $N[s]$ is homeomorphic to $N^{\prime}$. Suppose that the length of $\boldsymbol{s}$ on the maximal horoball of $T_{1}$ in int $N$ is at least $2 \pi+\epsilon$, for some $\epsilon>0$. Then, for any given $N^{\prime}$ and $\epsilon>0$, there is only a finite number of possibilities (up to isometry) for $N$ and $s$.

Remark 4.10. With the notation of Theorem 4.9, let $E$ denote the set of all slopes $s$ on $T_{1}$, such that $l(s) \leq 2 \pi$. It is a consequence of the Gromov-Thurston $2 \pi$ theorem that $E$ is finite. More specifically, the Gromov-Thurston theorem (a proof of which is found in [Bleiler and Hodgson 1996]) states that if $l(s)>2 \pi$, then $N[s]$ admits a negatively curved metric. But in Theorem 11 of [Bleiler and Hodgson 1996], Bleiler and Hodgson show that there can be at most 48 slopes on $T_{1}$ for which $N[s]$ admits no negatively curved metric. Thus, there can be at most 48 slopes on $T_{1}$ with length $\leq 2 \pi$.

Using Theorem 4.9 we will prove the following proposition which is a special case of Theorem 4.3 (compare possibility (b) on page 261):

Proposition 4.11. Let $K, K^{\prime}$ be knots such that $K \stackrel{m, L_{1}}{\longrightarrow} K^{\prime}$, where $L_{1}$ is a crossing circle for $K$ and $m>0$. Suppose that $N:=S^{3} \backslash \eta\left(K \cup L_{1}\right)$ is a hyperbolic manifold. Then there is a constant $b\left(K, K^{\prime}\right)$, depending only on $K, K^{\prime}$, such that $m \leq b\left(K, K^{\prime}\right)$.

Proof. We will apply Theorem 4.9 for the manifolds $N:=S^{3} \backslash \eta\left(K \cup L_{1}\right), N^{\prime}:=$ $S^{3} \backslash \eta\left(K^{\prime}\right)$ and the component $T_{1}:=\partial \eta\left(L_{1}\right)$ of $\partial N$. Let $s_{1}, \ldots, s_{m}$ be integers satisfying Definition 4.1. That is, for every nonempty $J \subset\{1, \ldots, m\}, N\left(s_{J}\right)$ is homeomorphic to $N^{\prime}$. By abusing the notation, for $r \in \mathbb{Z}$ we will write $l(r)$ for the length on $T_{1}$ of the slope represented by $\frac{1}{r}$. Also, as in the proof of Proposition 4.7, we will use $\Delta(r, t)$ to denote the geometric intersection on $T_{1}$ of the slopes represented by $\frac{1}{r}$ and $\frac{1}{t}$. Let $A(r, t)$ denote the area of the parallelogram in $\mathbb{R}^{2}$ spanned by the lifts of these slopes and let $A\left(T_{1}\right)$ denote the area of a fundamental domain of the torus $T_{1}$. It is known that $A\left(T_{1}\right) \geq \sqrt{3} / 2$ (see [Bleiler and Hodgson 1996]) and that $\Delta(r, t)$ is the quotient of $A(r, t)$ by $A\left(T_{1}\right)$. Thus, for every $r, t \in \mathbb{Z}$, we have

$$
l(r) l(t) \geq \Delta(r, t) \frac{\sqrt{3}}{2} .
$$

Let $\lambda>0$ denote the length of a meridian of $T_{1}$; in fact it is known that $\lambda \geq 1$. Assume to the contrary that no constant $b\left(K, K^{\prime}\right)$ as in the statement of the proposition 
exists. Then there exist infinitely many integers $s$ such that $N(s)$ is homeomorphic to $N^{\prime}$. Applying the preceding displayed inequality (to $l(s)$ and $\lambda$ ) we obtain

$$
l(s) \geq|s| \frac{\sqrt{3}}{2 \lambda} .
$$

Thus, for $|s| \geq(4 \pi \lambda+2 \lambda) / \sqrt{3}$, we have $l(s) \geq 2 \pi+1$. But then, for $\epsilon=1$, we have infinitely many integers such that $l(s) \geq 2 \pi+\epsilon$ and $N(s)$ is homeomorphic to $N^{\prime}$. Since this contradicts Theorem 4.9 the proof of the proposition is finished.

Next we turn our attention to the case where $N:=S^{3} \backslash \eta\left(K \cup L_{1}\right)$ is an atoroidal Seifert fibered space. Since $N$ is embedded in $S^{3}$ it is orientable. It is know that an orientable, atoroidal Seifert fibered space with two boundary components is either a cable space or a trivial torus bundle $T^{2} \times I$. Let us recall how a cable space is formed: Let $V^{\prime \prime} \subset V^{\prime} \subset S^{3}$ be concentric solid tori. Let $J$ be a simple closed curve on $\partial V^{\prime \prime}$ having slope $\frac{a}{b}$, for some $a, b \in \mathbb{Z}$ with $|b| \geq 2$. The complement $X:=V^{\prime} \backslash \operatorname{int} \eta(J)$ is a $\frac{a}{b}$-cable space. Topologically, $X$ is a Seifert fibered space over the annulus with one exceptional fiber of multiplicity $|b|$. We show the following:

Lemma 4.12. Let $K, K^{\prime}$ be knots such that $K \stackrel{m, L_{1}}{\longrightarrow} K^{\prime}$, where $L_{1}$ is a crossing circle for $K$ and $m>0$. Suppose that $N:=S^{3} \backslash \eta\left(K \cup L_{1}\right)$ is an irreducible, atoroidal Seifert fibered space. Then there is a constant $b\left(K, K^{\prime}\right)$ such that $m \leq b\left(K, K^{\prime}\right)$.

Proof. As discussed above, $N$ is either a cable space or a torus bundle $T^{2} \times I$. Note, however, that in a cable space the cores of the solid tori bounded in $S^{3}$ by the two components of $\partial N$ have nonzero linking number. Thus, since the linking number of $K$ and $L_{1}$ is zero, $N$ cannot be a cable space. Hence, we only have to consider the case where $N \cong T^{2} \times I$. Suppose $T_{1}=T^{2} \times\{1\}$ and $T_{2}:=\partial \eta(K)=T^{2} \times\{0\}$. By assumption there is a slope $s$ on $T_{1}$ such that the Dehn filling of $T_{1}$ along $s$ produces $N^{\prime}$. Now $s$ corresponds to a simple closed curve on $T_{2}$ that must compress in $N^{\prime}$. By Dehn's Lemma, $K^{\prime}$ must be the unknot. It follows that either $g(K)>g\left(K^{\prime}\right)$ or $K$ is the unknot. In the later case, we obtain that $L_{1}$ bounds a disc disjoint from $K$, contrary to our assumption that $N$ is irreducible. Thus, $g(K)>g\left(K^{\prime}\right)$ and the conclusion follows from Theorem 1.3.

The next result complements nicely Corollary 4.8; however, it is not needed for the proof of the main result. A reader eager to get to the proof of Theorem $4.3 \mathrm{can}$ move to the next page without loss of continuity.

Proposition 4.13. Let $K, K^{\prime}$ be nonisotopic hyperbolic knots. Suppose there exists a crossing circle $L_{1}$ for $K$ such that $K \stackrel{m, L_{1}}{\longrightarrow} K^{\prime}$, for some $m \geq 6$. Then, for given $K$ and $K^{\prime}$, there is only a finite number of possibilities for $m$ and for $L_{1}$ up to isotopy in the complement of $K$. 
Proof. As before, set $N:=S^{3} \backslash \eta\left(K \cup L_{1}\right), N^{\prime}:=S^{3} \backslash \eta\left(K^{\prime}\right)$ and let $D$ be a crossing disc for $L_{1}$. Since $K$ is not isotopic to $K^{\prime}, N$ is irreducible and $\partial$-irreducible.

\section{Claim. $N$ is atoroidal.}

Proof. Suppose that $N$ contains an embedded essential torus $T$ and let $V$ denote the solid torus bounded by $T$ in $S^{3}$. If $L_{1}$ cannot be isotoped to lie in int $V$ then $D \cap T$ contains a component whose interior in $D$ is pierced exactly once by $K$. This implies that $T$ is parallel to $\partial \eta(K)$ in $N$; a contradiction. Thus, $L_{1}$ can be isotoped to lie inside $V$. Now let $S$ be a Seifert surface of $K$ that is taut in $N$. After isotopy, $D \cap S$ is an $\operatorname{arc} \alpha$ that is essential on $S$. By Theorem 2.1, $S$ remains of minimum genus in at least one of $N^{\prime \prime}:=S^{3} \backslash \eta(K), N^{\prime}$. Assume $S$ remains of minimum genus in $N^{\prime}$; the other case is completely analogous. Since $K, K^{\prime}$ are hyperbolic $T$ becomes inessential in both of $N^{\prime \prime}, N^{\prime}$. But since $K, K^{\prime}$ are related by a generalized crossing change, either $T$ becomes boundary parallel in both of $N^{\prime \prime}$, $N^{\prime}$ or it becomes compressible in both of them. First suppose that $T$ is boundary parallel in both of $N^{\prime \prime}, N^{\prime}$ : Then it follows that the arc $\alpha$ is inessential on $S$ and $K$ is isotopic to $K^{\prime}$; a contradiction. Now suppose that $T$ is compressible in both of $N^{\prime \prime}, N^{\prime}$ : Then both of $K, K^{\prime}$ are inessential in $V$ and they can be isotoped to lie in a 3-ball $B \subset$ int $V$. By an argument similar to this in the proof of Lemma 4.6 we can conclude that $\alpha$, and thus $L_{1}$, can be isotoped to lie in $B$. But this contradicts the assumption that $T$ is essential in $N$ and finishes the proof of the claim.

To continue with the proof of the proposition, observe that the argument of the proof of Lemma 4.12 shows that if $N$ is a Seifert fibered space then $K^{\prime}$ is the unknot. But this is impossible since we assumed that $K^{\prime}$ is hyperbolic. Thus, by [Thurston 1979], $N$ is hyperbolic. Let $s_{1}, \ldots, s_{m}$ be integers that satisfy Definition 4.1 for $K, K^{\prime}$. Thus we have $2^{m}-1$ integers $s$, with $N(s)=N^{\prime}$. Now [Bleiler and Hodgson 1996] implies that we can have at most 48 integers so that the corresponding slopes have lengths $\leq 2 \pi$ on $T_{1}$. Since $m \geq 6$ we have $2^{m}-1>48$. Thus we have $k_{m}:=2^{m}-49>0$ integers $s$ such that $l(s)>2 \pi$ and $N(s)=N^{\prime}$. By Theorem 4.9 , there is only a finite number of possibilities (up to isometry) for $N$ and $s$. The proposition follows.

Remark 4.14. Proposition 4.13 implies Theorem 4.3, and thus also Theorem 1.2, if $K, K^{\prime}$ are hyperbolic.

We now turn to the proof of Theorem 4.3. We will need the following theorem, a special case of a result proved in [McCullough 2006].

Theorem 4.15 [McCullough 2006]. Let $M$ be a compact orientable 3-manifold, and let $C$ be a simple loop in $\partial M$. Suppose that $h: M \rightarrow M$ is a homeomorphism whose restriction to $\partial M$ is isotopic to a nontrivial power of a Dehn twist about $C$. Then $C$ bounds a disc in $M$. 
Recall also that for a compact orientable 3-manifold $M, \tau(M)$ denotes the cardinality of a Haken system of tori (page 262). In particular, $M$ is atoroidal if and only if $\tau(M)=0$.

Proof of Theorem 4.3. Let $K, K^{\prime}$ be knots and let $L_{1}$ be a crossing circle of $K$ such that $K \stackrel{m, L_{1}}{\longrightarrow} K^{\prime}$. As before we set $N:=S^{3} \backslash \eta\left(K \cup L_{1}\right)$ and $N^{\prime}:=S^{3} \backslash \eta\left(K^{\prime}\right)$. If $g(K)>g\left(K^{\prime}\right)$, by Theorem 1.3, we have $m \leq 3 g(K)-1$. Thus, in this case, we can take $b\left(K, K^{\prime}\right):=3 g(K)-1$ and Theorem 4.3 holds. Hence, we only have to consider that case that $g(K) \leq g\left(K^{\prime}\right)$.

Next we consider the complexity

$$
\rho=\rho\left(K, K^{\prime}, L_{1}\right):=\tau(N) .
$$

First, suppose that $\rho=0$, that is $N$ is atoroidal. Then $N$ is either hyperbolic or a Seifert fibered manifold [Thurston 1979]. In the former case, the conclusion of the theorem follows from Proposition 4.11; in the later case it follows from Lemma 4.12.

Assume now that $\tau(N)>0$; that is $N$ is toroidal. Suppose, inductively, that for every triple $K_{1}, K_{1}^{\prime}, L_{1}^{\prime}$, with $\rho\left(K_{1}, K_{1}^{\prime}, L_{1}^{\prime}\right)<r$, there is a constant $d=d\left(K_{1}, K_{1}^{\prime}\right)$ satisfying the following condition:

If $K_{1} \stackrel{m, L_{1}^{\prime}}{\longrightarrow} K_{1}^{\prime}$, then either $m \leq d$ or $L_{1}^{\prime}$ bounds an embedded disc in the complement of $K_{1}$.

Let $K, K^{\prime}, L_{1}$ be knots and a crossing circle for $K$ such that $K \stackrel{m, L_{1}}{\longrightarrow} K^{\prime}$ and $\rho\left(K, K^{\prime}, L_{1}\right)=r$. Let $s_{1}, \ldots, s_{m}$ be integers satisfying Definition 4.1 for $K, K^{\prime}$ and $L_{1}$. For every nonempty $J \subset\{1, \ldots, m\}$, let $N\left(s_{J}\right)$ be the 3-manifold obtained from $N$ by Dehn filling of $\partial \eta\left(L_{1}\right)$ with slope $\frac{1}{s_{J}}$. By assumption, $N^{\prime}=N\left(s_{J}\right)$. Assume, for a moment, that for some nonempty $J_{1} \subset\{1, \ldots, m\}, N\left(s_{J_{1}}\right)$ contains essential embedded tori of type II. Then Proposition 4.7 implies that either $m \leq 6$ or $L_{1}$ bounds an embedded disc in the complement of $K$. Hence, in this case, the conclusion of the theorem is true for $K, K^{\prime}, L_{1}$, with $b\left(K, K^{\prime}\right):=6$. Thus we may assume that, for every nonempty $J \subset\{1, \ldots, m\}, N\left(s_{J}\right)$ doesn't contain essential embedded tori of type II.

Claim. There exist knots $K_{1}, K_{1}^{\prime}$ and a crossing circle $L_{1}^{\prime}$ for $K_{1}$ such that

(1) $K_{1} \stackrel{m, L_{1}^{\prime}}{\longrightarrow} K_{1}^{\prime}$ and $\rho\left(K_{1}, K_{1}^{\prime}, L_{1}^{\prime}\right)<\rho\left(K, K^{\prime}, L_{1}\right)=r$, and

(2) if $L_{1}^{\prime}$ bounds an embedded disc in the complement of $K_{1}$ then $L_{1}$ bounds an embedded disc in the complement of $K$.

Assume the claim for the moment. By induction, there is $d=d\left(K_{1}, K_{1}^{\prime}\right)$ such that either $m \leq d$ or $L_{1}^{\prime}$ bounds a disc in the complement of $K_{1}$. Let $\mathcal{K}_{m}$ denote the set of all pairs of knots $K_{1}, K_{1}^{\prime}$ such that there exists a crossing circle $L_{1}^{\prime}$ for 
$K_{1}$ satisfying properties (1) and (2) of the claim. Define

$$
b=b\left(K, K^{\prime}\right):=\min \left\{d\left(K_{1}, K_{1}^{\prime}\right) \mid K_{1}, K_{1}^{\prime} \in \mathscr{K}_{m}\right\} .
$$

Clearly $b$ satisfies the conclusion of the statement of the theorem.

Proof of the claim. Let $T$ be an essential embedded torus in $N$. Since $T$ is essential in $N, T$ has to be knotted. Let $V$ denote the solid torus component of $S^{3} \backslash T$. Note that $K$ must lie inside $V$. For, otherwise $L_{1}$ must be geometrically essential in $V$ and thus it can't be the unknot. There are various cases to consider according to whether $L_{1}$ lies outside or inside $V$.

Case 1: Suppose that $L_{1}$ lies outside $V$ and it cannot be isotoped to lie inside $V$. Now $K$ is a nontrivial satellite with companion torus $T$. Let $D_{1}$ be a crossing disc bounded by $L_{1}$. Notice that if all the components of $D_{1} \cap T$ were either homotopically trivial in $D_{1} \backslash\left(D_{1} \cap K\right)$ or parallel to $\partial D_{1}$, then we would be able to isotope $L_{1}$ inside $V$, contrary to our assumption. Thus $D_{1} \cap T$ contains a component that encircles a single point of the intersection $K \cap D_{1}$. This implies that the winding number of $K$ in $V$ is one. Since $T$ is essential in $N$ we conclude that $K$ is composite, say $K:=K_{1} \# K_{2}$, and $T$ is the follow-swallow torus. Moreover, the generalized crossings realized by the surgeries on $L_{1}$ occur along a summand of $K$, say along $K_{1}$. By the uniqueness of prime decompositions of knots, it follows that there exists a (not necessarily nontrivial) knot $K_{1}^{\prime}$, such that $K^{\prime}=K^{\prime}{ }_{1} \# K_{2}$ and $K_{1} \stackrel{m, L_{1}}{\longrightarrow} K_{1}^{\prime}$. Set $N_{1}:=S^{3} \backslash \eta\left(K_{1} \cup L_{1}\right)$ and $N_{1}^{\prime}:=S^{3} \backslash \eta\left(K_{1}^{\prime}\right)$. Clearly, $\tau\left(N_{1}\right)<\tau(N)$. Thus, $\rho\left(K_{1}, K_{1}^{\prime}, L_{1}\right)<\rho\left(K, K^{\prime}, L_{1}\right)$ and part (1) of the claim has been proved in this case. To see part (2) notice that if $L_{1}$ bounds a disc $D$ in the complement of $K_{1}$, we may assume $D \cap K=\varnothing$.

Case 2: Suppose that $L_{1}$ can be isotoped to lie inside $V$. Now the link $K \cup L_{1}$ is a nontrivial satellite with companion torus $T$. We can find a standardly embedded solid torus $V_{1} \subset S^{3}$, and a 2-component link $\left(K_{1} \cup L_{1}^{\prime}\right) \subset V_{1}$ such that (i) $K_{1} \cup L_{1}^{\prime}$ is geometrically essential in $V_{1}$, (ii) $L_{1}^{\prime}$ is a crossing disc for $K_{1}$, and (iii) there is a homeomorphism $f: V_{1} \longrightarrow V$ such that $f\left(K_{1}\right)=K$ and $f\left(L_{1}^{\prime}\right)=L_{1}$ and $f$ preserves the longitudes of $V_{1}$ and $V$. In other words, $K_{1} \cup L_{1}^{\prime}$ is the model link for the satellite. Let $\mathscr{T}$ be a Haken system for $N$ containing $T$. We will assume that the torus $T$ is innermost; i.e. the boundary of the component of $N \backslash \mathscr{T}$ that contains $T$ also contains $\partial \eta(K)$. By twisting along $L_{1}$ if necessary, we may without loss of generality assume that $\bar{V}:=\overline{V \backslash\left(K \cup L_{1}\right)}$ is atoroidal. Then $\bar{V}_{1}:=$ $\overline{V_{1} \backslash\left(K_{1} \cup L_{1}^{\prime}\right)}$ is also atoroidal. For every nontrivial $J \subset\{1, \ldots, m\}$, let $K\left(s_{J}\right)$ denote the knot obtained from $K_{1}$ by performing $\frac{1}{S_{J}}$-surgery on $L_{1}^{\prime}$. By assumption the knots $f\left(K\left(s_{J}\right)\right)$ are all isotopic to $K^{\prime}$. 
Case 2a: There is a nonempty $J_{1} \subset\{1, \ldots, m\}$ such that $\partial V$ is compressible in $V \backslash f\left(K\left(s_{J_{1}}\right)\right)$. By Lemma 4.6, for every nonempty $J \subset\{1, \ldots, m\}, \partial V$ is compressible in $V \backslash f\left(K\left(s_{J}\right)\right)$. It follows that there is an embedded 3-ball $B \subset$ int $V$ such that (i) $f\left(K\left(s_{J}\right)\right) \subset \operatorname{int}(B)$, for every nonempty $J \subset\{1, \ldots, m\}$; and (ii) the isotopy from $f\left(K\left(s_{J_{1}}\right)\right)$ to $f\left(K\left(s_{J_{2}}\right)\right)$ can be realized inside $B$, for every $J_{1} \neq J_{2}$ as above. From this observation it follows that there is a knot $K_{1}^{\prime} \subset$ int $V_{1}$ such that

$$
f\left(K_{1}^{\prime}\right)=K^{\prime} \quad \text { and } \quad K_{1} \stackrel{m, L_{1}^{\prime}}{\longrightarrow} K_{1}^{\prime}
$$

in $V_{1}$. Set $N_{1}:=S^{3} \backslash \eta\left(K_{1} \cup L_{1}^{\prime}\right)$ and $N_{1}^{\prime}:=S^{3} \backslash \eta\left(K_{1}^{\prime}\right)$. Clearly, $\tau\left(N_{1}\right)<\tau(N)$. Hence, $\rho\left(K_{1}, K_{1}^{\prime}, L_{1}\right)<\rho\left(K, K^{\prime}, L_{1}\right)$ and the part (1) of the claim has been proved.

We will prove part (2) of the claim for Case 2 a together with the next case.

Case $2 b$ : For every nonempty $J \subset\{1, \ldots, m\}, f\left(K\left(s_{J}\right)\right)$ is geometrically essential in $V$. By Lemma 4.5, the conclusion of the claim is true if $K^{\prime}$ is composite. Thus, we may assume that $K^{\prime}$ is a prime knot. In this case, we claim that, for every nonempty $J_{1}, J_{2} \subset\{1, \ldots, m\}$, there is an orientation preserving homeomorphism $\phi: S^{3} \longrightarrow S^{3}$ such that $\phi(V)=V$ and $\phi\left(f\left(K\left(s_{J_{1}}\right)\right)\right)=f\left(K\left(s_{J_{2}}\right)\right)$. Since we assumed that $N\left(s_{J_{1}}\right), N\left(s_{J_{1}}\right)$ do not contain essential tori of type II, $T$ remains innermost in the complement of $f\left(K\left(s_{J_{1}}\right)\right), f\left(K\left(s_{J_{2}}\right)\right)$. By the uniqueness of the torus decomposition of knot complements [Jaco and Shalen 1979] or the uniqueness of satellite structures of knots [Schubert 1953], there is an orientation preserving homeomorphism $\phi: S^{3} \longrightarrow S^{3}$ such that $\phi(V) \cap V=\varnothing$ and $\bar{K}:=\phi\left(f\left(K\left(s_{J_{1}}\right)\right)\right)=$ $f\left(K\left(s_{J_{2}}\right)\right.$ ) (compare [Motegi 1993, Lemma 2.3]). Since $T$ is innermost in $\bar{V}$, we have $S^{3} \backslash$ int $V \subset \operatorname{int} \phi\left(S^{3} \backslash\right.$ int $\left.V\right)$ or $\phi\left(S^{3} \backslash\right.$ int $\left.V\right) \subset \operatorname{int}\left(S^{3} \backslash\right.$ int $\left.V\right)$. In both cases, by Haken's finiteness theorem, it follows that $T$ and $\phi(T)$ are parallel in the complement of $\bar{K}$. Thus after an ambient isotopy, leaving $\bar{K}$ fixed, we have $\phi(V)=V$. Let $h=f \circ \phi \circ f^{-1}: V_{1} \longrightarrow V_{1}$. Then $h$ preserves the longitude of $V_{1}$ up to a sign and $h\left(K\left(s_{J_{1}}\right)\right)=K\left(s_{J_{2}}\right)$. So, in particular, the knots $K\left(s_{J_{1}}\right)$ and $K\left(s_{J_{2}}\right)$ are isotopic in $S^{3}$. Let $K_{1}^{\prime}$ denote the knot type in $S^{3}$ of $\left\{K\left(s_{J}\right)\right\}_{J \subset\{1, \ldots, m\}}$. By our earlier assumptions, we have $K_{1} \stackrel{m, L_{1}^{\prime}}{\longrightarrow} K_{1}^{\prime}$. Set $N_{1}:=S^{3} \backslash \eta\left(K_{1} \cup L_{1}^{\prime}\right)$ and $N_{1}^{\prime}:=S^{3} \backslash \eta\left(K_{1}^{\prime}\right)$. Clearly, $\tau\left(N_{1}\right)<\tau(N)$. Thus part (1) of the claim has been proved also in this subcase.

We now prove part (2) of the claim for both subcases. Note that it is enough to show that if $L_{1}^{\prime}$ bounds an embedded disc, say $D^{\prime}$, in the complement of $K_{1}$ in $S^{3}$, then it bounds one inside $V_{1}$.

Let $D_{1}^{\prime} \subset V_{1}$ be a crossing disc bounded by $L_{1}^{\prime}$ and such that int $D^{\prime} \cap$ int $D_{1}^{\prime}=\varnothing$. Since $\partial V_{1}$ is incompressible in $V_{1} \backslash K_{1}$, after a cut and paste argument, we may assume that $E=D_{1}^{\prime} \cup\left(D \cap V_{1}\right)$ is a proper annulus whose boundary components are longitudes of $V_{1}$. 

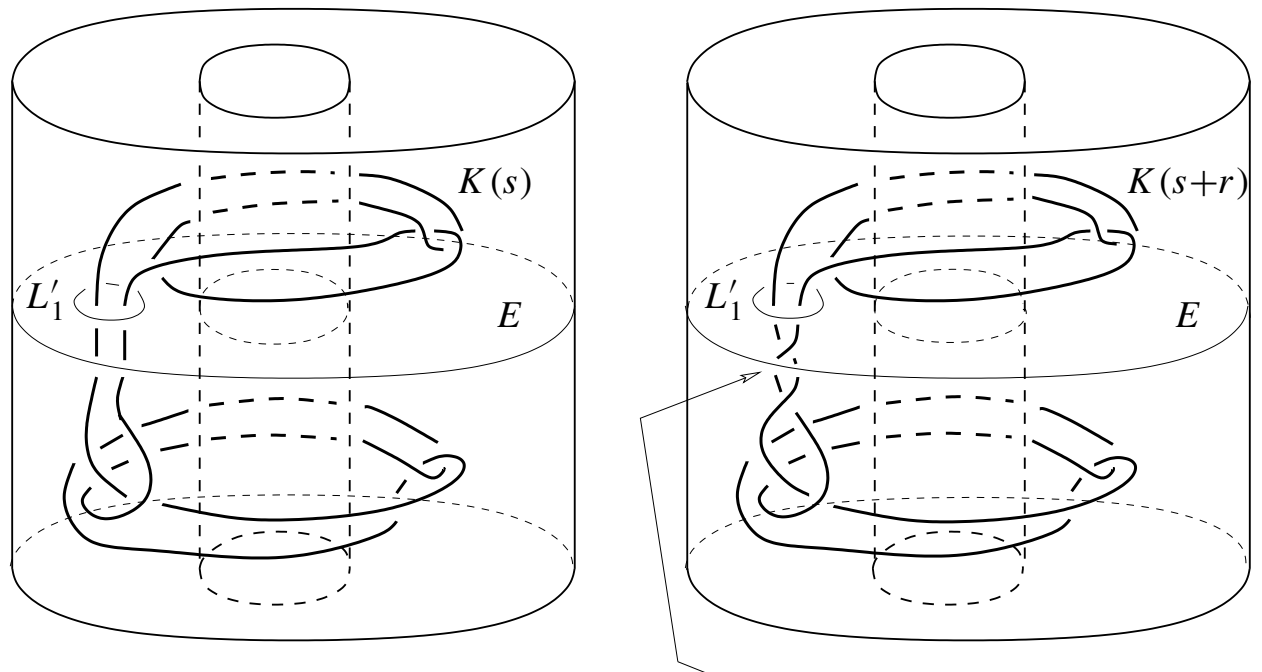

A twist of order $r$

Figure 4. The annulus $E$ contains the crossing circle $L_{1}^{\prime}$ and separates $V_{1}$ into solid tori $V_{1}^{\prime}$ (part above $E$ ) and $V_{1}^{\prime \prime}$ (part below $E$ ). In $V_{1}^{\prime \prime}$ the knots $K(s)$ and $K(s+r)$ differ by a twist of order $r$ along $D_{1}^{\prime}$.

By assumption, in both subcases, there exist nonzero integers $s, r$, such that $K(s)$ and $K(s+r)$ are isotopic in $S^{3}$. Here, $K(s)$ and $K(s+r)$ denotes the knots obtained from $K_{1}$ by a twist along $L_{1}^{\prime}$ of order $s$ and $s+r$ respectively. Let $\hat{h}: S^{3} \longrightarrow S^{3}$ denote the extension of $h: V_{1} \longrightarrow V_{1}$ to $S^{3}$. We assume that $\hat{h}$ fixes the core circle $C_{1}$ of the complementary solid torus of $V_{1}$. Since the 2sphere $D \cup D_{1}^{\prime}$ gives the same (possible trivial) connected sum decomposition of $K_{1}^{\prime}=K(s)=K(s+r)$ in $S^{3}$, we may assume that $\hat{h}(D)=D$ and $\hat{h}\left(D_{1}^{\prime}\right)=D_{1}^{\prime}$ up to an isotopy. During this isotopy of $\hat{h}, \hat{h}\left(C_{1}\right)$ and $\hat{h}\left(V_{1}\right)$ remain disjoint. So we may assume that at the end of the isotopy, we still have $\hat{h}\left(V_{1}\right)=V_{1}$. Thus, we can assume that $h(E)=E$.

The annulus $E$ cuts $V_{1}$ into two solid tori $V_{1}^{\prime}$ and $V_{1}^{\prime \prime}$. See Figure 4, where the solid torus above $E$ is $V_{1}^{\prime}$ and below $E$ is $V_{1}^{\prime \prime}$. We have either $h\left(V_{1}^{\prime}\right)=V_{1}^{\prime}$ and $h\left(V_{1}^{\prime \prime}\right)=V_{1}^{\prime \prime}$ or $h\left(V_{1}^{\prime}\right)=V_{1}^{\prime \prime}$ and $h\left(V_{1}^{\prime \prime}\right)=V_{1}^{\prime}$. In the case when $h\left(V_{1}^{\prime}\right)=V_{1}^{\prime}$ and $h\left(V_{1}^{\prime \prime}\right)=V_{1}^{\prime \prime}$, we may assume that $h \mid \partial V_{1}=\mathrm{id}$ and $h \mid E=\mathrm{id}$. Thus $K(s+r) \cap V_{1}^{\prime}=$ $K(s) \cap V_{1}^{\prime}$ and $K(s+r) \cap V_{1}^{\prime \prime}$ is equal to $K(s) \cap V_{1}^{\prime \prime}$ twisted by a twist of order $r$ along $L_{1}^{\prime}$. Let $M$ denote the 3-manifold obtained from $V_{1}^{\prime \prime} \backslash\left(V_{1}^{\prime \prime} \cap K(s)\right)$ by attaching a 2-handle to $\partial V_{1}^{\prime \prime} \cap E$ along $K(s) \cap V_{1}^{\prime \prime}$. Now $h \mid \partial M$ can be realized by a Dehn twist of order $r$ along $L_{1}^{\prime}$. By Theorem 4.15, $L_{1}^{\prime}$ must bound a disc in $M$. In order words, $L_{1}^{\prime}$ bounds a disc in $V_{1} \backslash K(s)$. This implies that $L_{1}^{\prime}$ bounds a disc in $V_{1} \backslash K_{1}$. 
In the case when $h\left(V_{1}^{\prime}\right)=V_{1}^{\prime \prime}$ and $h\left(V_{1}^{\prime \prime}\right)=V_{1}^{\prime}$, we may assume that $h \mid \partial V_{1}$ and $h \mid E$ are rotations of $180^{\circ}$ with an axis on $E$ passing through the intersection points of $D_{1}^{\prime}$ with $K(s)$ and $K(s+r)$. Thus $K(s+r) \cap V_{1}^{\prime}$ and $K(s) \cap V_{1}^{\prime \prime}$ differ by a rotation, and $K(s+r) \cap V_{1}^{\prime \prime}$ is equal to $K(s) \cap V_{1}^{\prime}$ twisted by a twist of order $r$ along $L_{1}^{\prime}$ followed by a rotation. Now we consider the 3-manifold $N$ obtained from $V_{1}^{\prime} \backslash\left(V_{1}^{\prime} \cap K(s)\right)$ by attaching a 2-handle to $\partial V_{1}^{\prime} \cap E$ along $K(s) \cap V_{1}^{\prime}$. As above we conclude that a Dehn twist of order $r$ along $L_{1}^{\prime}$ extends to $N$ and we complete the argument by applying Theorem 4.15.

\section{Applications and examples}

Applications to nugatory crossings. Recall that a crossing of a knot $K$ with crossing disc $D$ is called nugatory if $\partial D$ bounds a disc disjoint from $K$. This disc and $D$ bound a 2-sphere that decomposes $K$ into a connected sum, where some of the summands may be trivial. Clearly, changing a nugatory crossing doesn't change the isotopy class of a knot. An outstanding question is whether the converse is true:

Question 5.1 [Kirby 1997, Problem 1.58]. If a crossing change in a knot K yields a knot isotopic to $K$, is the crossing nugatory?

The answer is known to be yes when $K$ is the unknot [Scharlemann and Thompson 1989] or a 2-bridge knot [Torisu 1999]. Torisu conjectures that the answer is always yes. Our results in Section 5 yield the following corollary, which shows that an essential crossing circle of a knot $K$ can admit at most finitely many twists that do not change the isotopy type of $K$ :

Corollary 5.2. For a crossing of a knot $K$, with crossing disc $D$, let $K(r)$ denote the knot obtained by a twist of order $r$ along $D$. The crossing is nugatory if and only if $K(r)$ is isotopic to $K$ for all $r \in \mathbb{Z}$.

Proof. One direction of the corollary is clear. To obtain the other direction apply Theorem 4.3 for $K=K^{\prime}$.

In the view of this corollary, Question 5.1 is reduced to the following: In the same setting of Corollary 5.2, let $K_{+}:=K$ and $K_{-}:=K(1)$. If $K_{-}$is isotopic to $K_{+}$is it true that $K(r)$ is isotopic to $K$, for all $r \in \mathbb{Z}$ ?

Examples. Here we outline some methods that for every $n>0$ construct knots $K, K^{\prime}$ with $K \stackrel{n}{\longrightarrow} K^{\prime}$. It is known that given $n \in \mathbb{N}$ there exists a plethora of knots that are $n$-adjacent to the unknot. In fact, [Askitas and Kalfagianni 2002] provides a method for constructing all such knots. It is easy to see that given knots $K, K^{\prime}$ such that $K_{1}$ is $n$-adjacent to the unknot, the connected sum $K:=K_{1} \# K^{\prime}$ is $n$-adjacent to $K^{\prime}$. Clearly, if $K_{1}$ is nontrivial then $g(K)>g\left(K^{\prime}\right)$. To construct 
examples $K, K^{\prime}$ in which $K$ is not composite, at least in an obvious way, one can proceed as follows: For $n>0$ let $K_{1}$ be a knot that is $n$-adjacent to the unknot and let $V_{1} \subset S^{3}$ be a standard solid torus. We can embed $K_{1}$ in $V_{1}$ so that it has nonzero winding number and is $n$-adjacent to the core of $V_{1}$ inside $V_{1}$. Note that there might be many different ways of doing so. Now let $f: V_{1} \longrightarrow S^{3}$ be any embedding that knots $V_{1}$. Set $V:=f\left(V_{1}\right), K:=f\left(K_{1}\right)$ and let $K^{\prime}$ denote the core of $V$. By construction, $K \stackrel{n}{\longrightarrow} K^{\prime}$. Since $K_{1}$ has nonzero winding number in $V_{1}$ we have $g(K)>g\left(K^{\prime}\right)$ (see, for example, [Burde and Zieschang 1985]).

We will say that two ordered pairs of knots $\left(K_{1}, K_{1}^{\prime}\right),\left(K_{2}, K_{2}^{\prime}\right)$ are isotopic if and only if $K_{1}$ is isotopic to $K_{2}$ and $K_{1}^{\prime}$ is isotopic to $K_{2}^{\prime}$. From our discussion above we obtain:

Proposition 5.3. For every $n \in \mathbb{N}$ there exist infinitely many nonisotopic pairs of knots $\left(K, K^{\prime}\right)$ such that $K \stackrel{n}{\longrightarrow} K^{\prime}$ and $g(K)>g\left(K^{\prime}\right)$.

Remark 5.4. We don't know of any examples of knots $\left(K, K^{\prime}\right)$ such that $K \stackrel{n}{\longrightarrow} K^{\prime}$ and $g(K) \leq g\left(K^{\prime}\right)$. In fact the results of [Kalfagianni 2006], and further examples constructed in [Torisu 2006], prompt the following question: Is it true that if $K \stackrel{n}{\longrightarrow} K^{\prime}$ for some $n>1$, then either $g(K)>g\left(K^{\prime}\right)$ or $K$ is isotopic to $K^{\prime}$ ?

\section{Acknowledgments}

We thank Tao Li, Katura Miyazaki and Ying-Qing Wu for their interest in this work and for their helpful comments on an earlier version of the paper. We thank Darryl McCullough for his comments and his result [2006] about homeomorphisms of 3-manifolds needed for the proof the main result of this paper. We also thank Ian Agol, Steve Bleiler, Dave Gabai, Marc Lackenby, Marty Scharlemann and Oleg Viro for useful conversations or correspondence. Finally, we thank the referee for a very thoughtful and careful review that has led to significant improvements in exposition.

\section{References}

[Askitas and Kalfagianni 2002] N. Askitas and E. Kalfagianni, "On knot adjacency”, Topology Appl. 126:1-2 (2002), 63-81. MR 2004f:57008 Zbl 1012.57018

[Bleiler and Hodgson 1996] S. A. Bleiler and C. D. Hodgson, "Spherical space forms and Dehn filling”, Topology 35:3 (1996), 809-833. MR 97f:57007 Zbl 0863.57009

[Burde and Zieschang 1985] G. Burde and H. Zieschang, Knots, Studies in Mathematics 5, de Gruyter, Berlin, 1985. MR 87b:57004 Zbl 0568.57001

[Cooper and Lackenby 1998] D. Cooper and M. Lackenby, "Dehn surgery and negatively curved 3-manifolds", J. Differential Geom. 50:3 (1998), 591-624. MR 2000g:57030 Zbl 0931.57014

[Gabai 1987] D. Gabai, "Foliations and the topology of 3-manifolds, II", J. Differential Geom. 26:3 (1987), 461-478. MR 89a:57014a Zbl 0627.57012 
[Gordon 1998] C. M. Gordon, "Boundary slopes of punctured tori in 3-manifolds", Trans. Amer. Math. Soc. 350:5 (1998), 1713-1790. MR 98h:57032 Zbl 0896.57011

[Hempel 1976] J. Hempel, 3-Manifolds, Ann. of Math. Studies 86, Princeton University Press, Princeton, NJ, 1976. MR 54 \#3702 Zbl 0345.57001

[Howards and Luecke 2002] H. Howards and J. Luecke, "Strongly $n$-trivial knots", Bull. London Math. Soc. 34:4 (2002), 431-437. MR 2003c:57008 Zbl 1027.57004

[Jaco and Shalen 1979] W. H. Jaco and P. B. Shalen, Seifert fibered spaces in 3-manifolds, Mem. Amer. Math. Soc. 220, Amer. Math. Soc., Providence, 1979. MR 81c:57010 Zbl 0415.57005

[Kalfagianni 2004] E. Kalfagianni, "Alexander polynomial, finite type invariants and volume of hyperbolic knots”, Algebr. Geom. Topol. 4 (2004), 1111-1123. MR 2005k:57017 Zbl 1078.57014

[Kalfagianni 2006] E. Kalfagianni, "Crossing changes in fibered knots", preprint, 2006. Available at math.GT/0610440

[Kalfagianni and Lin 2004a] E. Kalfagianni and X.-S. Lin, "Knot adjacency and fibering", preprint, 2004. To appear in Trans. Amer. Math. Soc. Available at math.GT/0403026

[Kalfagianni and Lin 2004b] E. Kalfagianni and X.-S. Lin, "Knot adjacency and satellites", Topology Appl. 138:1-3 (2004), 207-217. MR 2005e:57019 Zbl 1045.57002

[Kirby 1997] R. Kirby, "Problems in low-dimensional topology", pp. 35-473 in Geometric topology (Athens, GA, 1993), vol. 2, edited by W. H. Kazez, AMS/IP Studies in Advanced Mathematics 2.2, American Mathematical Society, Providence, RI, 1997. MR 98f:57001 Zbl 0882.00041

[McCullough 2006] D. McCullough, "Homeomorphisms which are Dehn twists on the boundary", Algebr. Geom. Topol. 6 (2006), 1331-1340. MR MR2253449

[Motegi 1993] K. Motegi, "Knotting trivial knots and resulting knot types", Pacific J. Math. 161:2 (1993), 371-383. MR 94h:57012 Zbl 0788.57004

[Ng and Stanford 1999] K. Y. Ng and T. Stanford, "On Gusarov's groups of knots", Math. Proc. Cambridge Philos. Soc. 126:1 (1999), 63-76. MR 2000d:57007 Zbl 0961.57006

[Ohyama 1990] Y. Ohyama, "A new numerical invariant of knots induced from their regular diagrams”, Topology Appl. 37:3 (1990), 249-255. MR 92a:57009 Zbl 0724.57006

[Scharlemann and Thompson 1989] M. Scharlemann and A. Thompson, "Link genus and the Conway moves”, Comment. Math. Helv. 64:4 (1989), 527-535. MR 91b:57006 Zbl 0693.57004

[Schubert 1953] H. Schubert, "Knoten und Vollringe”, Acta Math. 90 (1953), 131-286. MR 17,291d Zbl 0051.40403

[Thurston 1979] W. P. Thurston, "The geometry and topology of three-manifolds", lecture notes, Princeton University, 1979, Available at http://msri.org/publications/books/gt3m.

[Thurston 1986] W. P. Thurston, "A norm for the homology of 3-manifolds", Mem. Amer. Math. Soc. 59:339 (1986), i-vi and 99-130. MR 88h:57014 Zbl 0585.57006

[Torisu 1999] I. Torisu, "On nugatory crossings for knots", Topology Appl. 92:2 (1999), 119-129. MR 2000b:57015 Zbl 0926.57004

[Torisu 2006] I. Torisu, "On 2-adjacency relations of 2-bridge knots and links", preprint, 2006.

[Vassiliev 1990] V. A. Vassiliev, "Cohomology of knot spaces", pp. 23-69 in Theory of singularities and its applications, Adv. Soviet Math. 1, Amer. Math. Soc., Providence, RI, 1990. MR 92a:57016 Zbl 0727.57008

Received April 6, 2005. Revised June 26, 2006. 


\section{EFSTRATIA KALFAGIANNI}

DEPARTMENT OF MATHEMATICS

WELLS HALL

Michigan State UnIVERSITY

EAST LANSING, MI 48824

UNITED STATES

kalfagia@math.msu.edu

http://www.math.msu.edu/ kalfagia

XIAO-SONG LIN

DEPARTMENT OF MATHEMATICS

UNIVERSITY OF CALIFORNIA, RIVERSIDE

RIVERSIDE, CA 92521-0135

UNITED STATES

xl@math.ucr.edu

http://math.ucr.edu/ xl 